\title{
Queixa escolar: uma revisão crítica da produção científica nacional
}

\author{
Maria Virgínia Machado Dazzani \\ Universidade Federal da Bahia - BA \\ Eliseu de Oliveira Cunha \\ Universidade Federal da Bahia - BA \\ Polyana Monteiro Luttigards \\ Universidade Federal da Bahia - BA \\ Patrícia Carla Silva do Vale Zucoloto \\ Faculdade Ruy Barbosa - BA \\ Gilberto Lima dos Santos \\ Universidade Estadual da Bahia - BA
}

\begin{abstract}
Resumo
Este trabalho teve como objetivo revisar a produção científica brasileira publicada entre 2002 e 2012 sobre "queixa escolar" e analisar criticamente as suas principais características e os aspectos associados à sua produção. Foi realizada uma busca sistemática em três bases de dados bibliográficos (SCIELO, PEPSIC e Biblioteca Digital Brasileira de Teses e Dissertações), com as palavras-chave queixa escolar e queixas escolares. Foram encontradas, inicialmente, 35 produções, e após uma pré-análise, restaram 21 trabalhos, sendo treze artigos e oito dissertações de mestrado. Os estudos foram analisados considerando-se suas principais características e aspectos relacionados à produção da queixa escolar. Os resultados apontam que o referencial teórico predominante entre os estudos foi a Psicologia Sócio-histórica e a Psicologia Escolar Crítica, com uma abordagem dialética e ampliada da queixa escolar, considerando as dimensões históricas, socioculturais, institucionais e político-econômicas da rede de relações da qual ela emerge.
\end{abstract}

Palavras-chave: Revisão de literatura; psicologia educacional; fracasso escolar.

\section{School complaint: a critical review of the national scientific literature}

\begin{abstract}
In this work we review Brazilian scientific publications between 2002 and 2012 on School Complaints, in order to critically analyze their main features and the aspects which are associated with generating a school complaint. We developed a systematic search in three bibliographic databases (SCIELO PEPSIC and the Brazilian Digital Library of Theses and Dissertations) using the two key words in Portuguese 'queixa escolar' and 'queixas escolares'. Initially 35 publications were found and, after pre-analysis, 21 papers remained: 13 articles and 8 master's theses. The studies were analyzed, taking their main characteristics and aspects related to generating a School Complaint in account. The results show that the predominating theoretical reference frame in the studies was Socio-historical Psychology and Critical School Psychology, with a dialectic and wide approach of school complaints, considering the historical, socio-cultural, institutional and political-economic dimensions of the social network in which the school complaints emerge.
\end{abstract}

Keywords: Literature review; educational psychology; academic failure.

\section{Queja escolar: una revisión crítica de la producción científica nacional}

\section{Resumen}

Este estudio tuvo como objetivo revisar la producción científica brasileña publicada entre 2002 y 2012 sobre "queja escolar" y analizar críticamente sus principales características y los aspectos asociados a su producción. Fue realizada una búsqueda sistemática en tres bases de datos bibliográficos (SCIELO, PEPSIC y Biblioteca Digital Brasileña de Tesis y Disertaciones), con las palabras-clave queja escolar y quejas escolares. Fueron encontradas, inicialmente, 35 producciones, y tras un pre-análisis, restaron 21 trabajos, siendo trece artículos y ocho disertaciones de maestría. Los estudios fueron analizados considerándose sus principales características y aspectos relacionados a la producción de la queja escolar. Los resultados apuntan que el referencial teórico predominante entre los estudios fue la Psicología Socio-histórica y la Psicología Escolar Crítica, con un abordaje dialéctico y ampliada de la queja escolar, considerando las dimensiones históricas, socioculturales, institucionales y político-económicas de la red de relaciones de la cual ella emerge.

Palabras-clave: revisión de literatura; Psicología Educacional; fracaso escolar. 


\section{Introdução}

Nos últimos anos o tema da queixa escolar tem recebido grande atenção da comunidade acadêmica, tanto no campo da Educação quanto no da Psicologia. No seu entorno há a proliferação de uma rica literatura (Souza, 2000; Scortegagna, \& Levandowski, 2004; Marçal, \& Silva, 2006; Neves, \& Marinho-Araujo, 2006; Braga, \& Morais, 2007; Souza, 2007; Nakamura, Lima, Tada, \& Junqueira, 2008; Bray, \& Leonardo, 2011; Marinho-Araujo, \& Guzzo, 2011).

Frequentemente a queixa escolar, sobretudo no interior da escola pública, tem demandado a atenção e a intervenção de serviços de saúde e, consequentemente, a atuação de profissionais como psicólogos, pedagogos, psiquiatras, neurologistas, terapeutas ocupacionais e fonoaudiólogos. Por "queixa escolar" entendem-se as demandas formuladas por pais, professores e coordenadores pedagógicos acerca de dificuldades e problemas enfrentados por estudantes no ambiente escolar.

De acordo com Cabral e Sawaya (2001), dois fenômenos merecem uma atenção especial: 1- é um fato muitíssimo eloquente que entre 50 e $70 \%$ das crianças e adolescentes encaminhadas aos serviços públicos de saúde apresentam queixas relativas especificamente a dificuldades de aprendizagem ou problemas de comportamento na escola; 2- é comum ouvir relatos de professores que afirmam que os alunos são indisciplinados, agressivos, têm dificuldades de aprendizagem, que a precariedade das condições de vida atrapalha o desempenho escolar, que os pais não têm interesse pela educação dos filhos e que, por fim, os estudantes não estão interessados pelo que é ensinado na escola.

Esses dois fenômenos sugerem que a queixa escolar localiza no estudante uma causa interna dos problemas de desempenho escolar. Seriam razões intrapsíquicas ou orgânicas que causariam os problemas de aprendizagem e disciplinares. Neste sentido, a atenção à queixa tem sido prioritariamente centrada no educando, numa perspectiva clínica, baseada frequentemente em avaliação psicométrica e médica, seguindo um modelo médico-fisicalista (Machado, 1997; Moysés, 1998; Cabral, \& Sawaya, 2001; Neves, \& Marinho-Araujo, 2006; Souza, 2007).

No outro lado da moeda haveria também causas ligadas às condições culturais, sociais e econômicas das famílias - principalmente o nível de escolaridade dos pais - que poderiam ocasionar uma baixa adesão ao processo escolar dos filhos e por consequência, também o desempenho do estudante (Patto, 1990; Bock, \& Aguiar, 2003; Nogueira, 2004; Nogueira, 2006; Nogueira, \& Abreu, 2004; Dazzani, \& Faria, 2009); ou seja, em torno do discurso da "queixa" não é incomum a emergência de um discurso que, de certo modo, patologiza ora a criança ora a pobreza das famílias.

\section{A Emergência da "queixa escolar"}

Podemos identificar na queixa escolar a centralidade dos problemas de escolarização e da não aprendizagem
(Bray \& Leonardo, 2011). Neste sentido, encontramos em Scortegagna e Levandowski (2004) a noção de queixa escolar como encaminhamento para atendimento especializado de crianças que protagonizam problemas escolares ou supostos distúrbios de comportamento e de aprendizagem.

$\mathrm{Na}$ literatura pertinente ao tema nota-se que a expressão e a produção da queixa escolar têm-se constituído como um fenômeno que mantém as mesmas características ao longo dos últimos anos e nas diferentes regiões do Brasil (Machado, 1997; Moysés, 1998, Cabral \& Sawaya, 2001; Neves \& Marinho-Araujo, 2006; Souza, 2007). Scortegagna e Levandowski (2004) fazem referência a alguns estudos realizados na década de 1990 que já apresentavam a compreensão de que o enfoque psicológico é insuficiente para avaliar o fracasso escolar, fenômeno cuja complexidade comporta dimensões pedagógicas e sociais (Patto, 1990; Machado, 1997; Moysés, 1998).

Esses estudos seriam, então, a expressão de um ponto de inflexão ocorrido no âmbito da Psicologia Escolar e Educacional no decorrer da década de 1980, quando uma visão crítica sobre os processos escolares começava a ser construída. Novas formas de analisar esses processos puseram em pauta, inclusive, o questionamento da formação do psicólogo e da adequação do instrumental até então utilizado por este na avaliação dos referidos fenômenos e na subsequente intervenção.

Referindo-se a estudos realizados ao longo da década de 2000, Bray e Leonardo (2011) traçam um cenário muito semelhante àquele que identificamos nos estudos de Scortegagna e Levandowski (2004) referente à década de 1990; ou seja, predomina ainda uma visão individualizante do fracasso escolar, que é centrado principalmente no aluno, mas, também, às vezes, na família ou no professor. De certo modo, parece que o próprio ato de "encaminhar" uma criança já traz uma interpretação na forma de "diagnóstico" que é produzido pela escola. Scortegagna e Levandowski (2004), ao avaliarem as queixas presentes nos encaminhamentos, inseriram a maioria delas em três grandes categorias, a saber: problemas de aprendizagem, problemas de comportamento e problemas emocionais.

De modo geral, os achados de Scortegagna e Levandowski (2004), Nakamura e cols. (2008), Zibetti e cols. (2010) e Bray e Leonardo (2011) são convergentes no sentido de indicarem a manutenção de práticas de produção, encaminhamento e atendimento às queixas escolares que se mostram individualizantes e estigmatizantes. Isso, ademais, revela que há dificuldades epistêmicas na relação estabelecida entre a escola e a família, pois as queixas são produzidas numa rede de relações que inclui a criança, a escola e a família e o contexto sociocultural e histórico em que estas estão situadas.

Nesse contexto, este trabalho teve como objetivo revisar a produção científica brasileira sobre queixa escolar publicada entre 2002 e 2012, a fim de analisar criticamente as suas principais características e os aspectos associados à produção da queixa escolar. 


\section{Método}

\section{Fonte}

Foram identificados todos os trabalhos publicados no período de 10 anos (2002-2012) em artigos de revistas indexadas nas bases de dados Scielo e Pepsic, além de teses e dissertações disponibilizadas na Biblioteca Digital Brasileira de Teses e Dissertações sobre queixa escolar.

\section{Procedimento}

Buscaram-se trabalhos acadêmicos em cujo título houvesse a expressão queixa escolar ou queixas escolares. De início, os resumos de todas as publicações encontradas no período de 2004 a 2011 foram lidos cuidadosamente, e assim se conseguiu organizar os trabalhos de acordo com a base de dados e o tipo de publicação. Posteriormente, foi feita uma seleção no material obtido, e das 35 produções encontradas foram subtraídas 14 (cinco resenhas de livros, três trabalhos indexados em outra base de dados e seis artigos que apresentavam uma significativa distância em relação à queixa escolar enquanto fenômeno educativo e psicossocial). Restaram, assim, 21 trabalhos (treze artigos e oito dissertações de mestrado), dos quais 17 são estudos empíricos e quatro são estudos teóricos. Em seguida, as 21 publicações foram lidas na íntegra, para se fazer uma análise crítica de suas principais características e dos processos associados à produção da queixa escolar.

\section{Resultados e discussão}

Para a apresentação e discussão dos resultados encontrados nos 21 trabalhos publicados, foram construídas duas categorias. A primeira, intitulada "Principais características dos trabalhos", contém duas subcategorias: "Abordagem teórico-metodológica" e "Procedência geográfica". A segunda categoria, "Aspectos relacionados à produção da queixa escolar", apresenta tópicos que foram destacados nesta revisão por estarem significativamente presentes nas publicações. Esses tópicos foram apresentados na forma de seis subcategorias, a saber: 1- Perfil dos alunos identificados com demandas de queixa escolar; 2- Formação e atuação profissional deficitária; 3- falta de contato com a escola; 4- Culpabilização do aluno e de sua família; 5- A Psicologia como mantenedora do fracasso escolar; 6- A Escola como produtora do fracasso escolar.

\section{Principais características dos trabalhos}

\section{Abordagem teórico-metodológica \\ Com a adoção do critério de similaridades de refe-} rencial teórico e técnicas de análise de dados, foi possível categorizar os trabalhos em três abordagens teórico-metodológicas distintas: Sócio-histórica/crítica, Comportamental/ cognitiva e Filosófica/foucaultiana.

$\mathrm{Na}$ primeira abordagem foram categorizados treze trabalhos, os quais tiveram o protagonismo teórico da Psicologia Sócio-histórica e da Psicologia Escolar Crítica. A abordagem Sócio-histórica diz respeito àqueles trabalhos desenvolvidos a partir da obra de L. S. Vigotski, os quais têm conduzido pesquisadores a uma compreensão historicizada do psiquismo humano "para além de explicações baseadas em modelos mecanicistas ou modelos organicistas de desenvolvimento" (Facci, 2004, p. 65).

A perspectiva crítica está relacionada à ruptura epistemológica com concepções adaptativas de Psicologia e com um intenso movimento na área pela busca de referenciais teórico-metodológicos no campo da Psicologia da Educação que compreendam, segundo as palavras de Souza (2009):

a) os fenômenos escolares enquanto produtos do processo de escolarização, constituídos pelas dimensões institucional, pedagógica e relacional; b) o desenvolvimento humano e a aprendizagem enquanto processos inseparáveis, articulando as dimensões biológica, psicológica e histórica dos indivíduos; c) a necessidade de construir instrumentos psicológicos de aproximação e de conhecimento da realidade que permitam compreender a complexidade dos fenômenos educativos; d) a consideração da dimensão educativa no trabalho psicológico (p. 180).

Das treze publicações, nove foram artigos (Scortegagna \& Levandowski, 2004; Souza, 2005; Marçal, \& Silva, 2006; Neves, \& Marinho-Araujo, 2006; Souza, 2006; Trautwein, \& Nébias, 2006; Braga, \& Morais, 2007; Nakamura, \& cols., 2008; Bray, \& Leonardo, 2011) e quatro eram dissertações de mestrado (Pereira, 2004; Marçal, 2005; Dias, 2008; Rosa, 2011). Os principais instrumentos de pesquisa utilizados nestes trabalhos foram prontuários de atendimento psicológico, questionários com perguntas abertas, entrevistas semidirigidas e análise documental. Foi consensual, entre os autores situar o fracasso e, consequentemente, a queixa escolar como um fenômeno complexo, decorrente de uma rede de relações que perpassa por dimensões históricas, sociais, institucionais e político-econômicas.

Na segunda abordagem foram elencadas sete produções, as quais tiveram o protagonismo teórico das abordagens psicológicas comportamental e cognitiva, sendo quatro artigos (Marturano, Toller, \& Elias, 2005; D'Avila-Bacarji, Marturano, \& Elias, 2005; Elias, \& Marturano, 2005; Ferreira, Conte, \& Marturano; 2011) e três dissertações de mestrado (Araújo, 2006; Panaia, 2007; Barros, 2008). Foi consensual a submissão dos dados destes trabalhos à análise quantitativa e qualitativa, e os estudos foram, em geral, experimentais, com a aplicação de testes psicológicos, questionários objetivos e outros instrumentos psicométricos, visando avaliar, quantificar e comparar o desempenho dos participantes em comportamentos e características psicológicas concernentes à queixa escolar, com vista a analisar e identificar as variáveis com as quais se encontram relacionadas. 
Por fim, na abordagem filosófica/foucaultiana foi identificada apenas a dissertação de Bernardes (2008), estudo teórico no qual a autora faz uma análise da queixa escolar à luz das ideias do filósofo francês Michel Foucault.

\section{Procedência geográfica}

Os estudos mapeados são procedentes de apenas seis estados brasileiros. Dos 21 trabalhos selecionados, apenas um foi desenvolvido na Região Norte do Brasil, no Estado de Rondônia. Da Região Centro-Oeste foram publicados dois estudos, ambos do Distrito Federal, e igual número de trabalhos encontrados procediam da Região Sul, sendo um do Paraná e um do Rio Grande do Sul. Da Região Sudeste, identificamos quinze trabalhos publicados, abarcando apenas os Estados de Minas Gerais, com três publicações, e de São Paulo, com doze. Assim, a maioria dos trabalhos foi produzida no Estado de São Paulo. Não foi encontrado nenhum trabalho produzido na Região Nordeste e em um dos artigos encontrados não se especificou a procedência.

\section{Aspectos relacionados à produção da queixa escolar}

\section{Perfil dos alunos identificados com demandas de queixa escolar}

Percebeu-se, com esta revisão, que a maioria dos autores não contemplou na sua pesquisa características como faixa etária, período escolar ou origem social dos estudantes. Uma hipótese para este tratamento pode ser a de que muitos autores no campo da Psicologia Escolar Crítica tendem a não atribuir ao estudante a culpa (na verdade, a responsabilidade) pelo seu fracasso escolar; contudo, embora seja necessário relativizar a responsabilização pelo insucesso na escola, é indispensável considerar as informações concernentes à vida da criança encaminhada, principalmente dados sociodemográficos, para se construir uma visão ampliada da queixa escolar.

Os poucos dados levantados por alguns autores relativos ao perfil do estudante apontam um número maior de encaminhamentos no período do Ensino Fundamental, entre o $3^{\circ}$ e $5^{\circ}$ anos. É neste período que as crianças são mais frequentemente encaminhadas aos diferentes serviços de atenção à infância, incluindo os Centros de Atenção Psicossocial e Serviços de Psicologia, entre outros. No que tange à faixa etária principal dos encaminhamentos, tanto Souza (1989) quanto Marçal (2005) indicam uma faixa semelhante - de cinco a catorze anos e de cinco a treze anos, respectivamente.

Os artigos e dissertações que elucidam as diferenças entre gênero apresentam um número superior de casos de encaminhamentos de crianças/adolescentes do sexo masculino, com queixas relacionadas a "problemas de conduta". Apenas um dos textos encontrados nesta revisão (Nakamura \& cols., 2008) explicita as diferenças entre rede pública e privada de ensino. No estudo, do total de estudantes cujos dados foram acessados por meio de prontuários, 91\% são procedentes da rede pública.

\section{Formação e atuação profissional deficitária}

Alguns autores denunciam a significativa deficiência da formação do psicólogo, alertando para o fato de que nos cursos predominam as abordagens clínica e individual. Estas abordagens, em geral, negligenciam as dimensões sociais, políticas e históricas dos processos de aprendizagem. Neves e Marinho-Araujo (2006), por exemplo, apontam uma recorrente transposição acrítica dos modelos da psicologia clínica no atendimento à queixa escolar. Segundo Araújo (2006), a situação não é diferente quanto à formação do professor. A autora identificou deficiências nos cursos de graduação em Pedagogia no que concerne ao atendimento à queixa escolar.

No estudo de Marçal e Silva (2006), 90\% dos psicólogos entrevistados queixaram-se de inaptidão profissional para o atendimento de queixas escolares em serviços públicos, julgando necessária uma pós-graduação na área para melhor capacitá-los. Foi recorrente entre os autores o alerta para a urgente necessidade de repensar e reformular as teorias e práticas pedagógicas e psicológicas relacionadas aos problemas escolares (Souza, 2005).

\section{Ausência de contato com a escola}

Os estudos revelaram que os profissionais de Psicologia que atendem alunos encaminhados com queixa escolar não mantêm contato com a escola, ou o fazem de modo superficial (Araújo, 2006; Braga, \& Morais, 2007; Dias, 2008; Marçal, 2005; Marçal, \& Silva, 2006; Nakamura, \& cols., 2008; Rosa, 2011; Souza, 2005). Geralmente os atendimentos de queixa escolar são realizados em locais como clínicas-escola, ambulatórios de saúde mental e consultórios particulares.

Rosa (2011) observou a inexistência de contato entre a escola e a psicóloga. Esta última, no máximo, analisa o material escolar dos alunos, desconhecendo o contexto educacional ao qual estão vinculados. Dias (2008) identificou que o contato com a escola, quando ocorre, é feito por meio de relatórios enviados pelos educadores que apenas ratificam a queixa que motivou o encaminhamento. De maneira semelhante, Nakamura e cols. (2008) concluíram que os psicólogos não se dirigem à escola para conversar com os demais atores escolares, nem procuram compreender quais as condições estruturais do local da queixa.

Nos estudos de Marçal e Silva (2006) verificou-se que $93,7 \%$ dos psicólogos nunca entraram em contato com a escola. Nas entrevistas relatadas por Braga e Morais (2007) constatou-se que em apenas $1 \%$ dos casos de encaminhamento por queixa escolar foi realizada entrevista com o professor, e na amostra estudada por Araújo (2006), apenas $18,3 \%$ dos psicólogos falaram sobre o trabalho em parceria com os professores. Dentre os psicólogos entrevis- 
tados por Marçal (2005), somente $25 \%$ consideraram importante contatar a escola, alguns alegaram falta de tempo e outros relataram que a escola não teve interesse em fazer uma parceria.

\section{Culpabilização do aluno e sua família}

Em geral, os estudos apontam uma característica comum entre alguns profissionais que lidam com estudantes com histórico de queixa escolar, tanto em escolas quanto em serviços de atendimento, em relação aos eventos que desencadeiam a queixa: há uma tendência a responsabilizar o aluno e a sua família pelo fracasso escolar - ou seja, há um discurso institucionalizado que estabelece uma relação necessária entre, de um lado, o fenômeno fracasso escolar, e do outro, a atuação direta da família e as características psicológicas do indivíduo. Por essa razão, para entendermos corretamente o sentido e as ocorrências da queixa escolar, é indispensável investigarmos atentamente não só os atores primários (o aluno, a família e o professor), mas também o contexto social e institucional implicado na queixa, a saber: a) o processo de formação do saber técnico-institucional que já se encontra no modelo de formação profissional de professores, pedagogos e psicólogos; b) a estrutura das relações de poder-saber que se encontra nas escolas e nos serviços de atendimento. Com isso, poderemos superar o discurso de patologização e culpabilização dos atores escolares.

Em rigor, o discurso hegemônico hoje adota um modelo extraído da Biologia e da Psicologia, mas ignora as dimensões institucionais, históricas, sociais e político-econômicas que perpassam esses fenômenos. Isso faz com que a dimensão propriamente pedagógica do fracasso se perca e questões que estão diretamente associadas à dimensão política e social sejam ignoradas (Bray, \& Leonardo, 2011; Nakamura, \& cols., 2008; Neves, \& Marinho-Araujo, 2006; Scortegagna, \& Levandowski, 2004; Souza, 2005). Isso não significa que a Biologia e a Psicologia sejam dispensáveis para o entendimento do processo de aprendizagem: a questão diz respeito ao poder explicativo e às consequências pedagógico-sociais do discurso baseado exclusivamente nesse modelo.

No tocante à questão, nos artigos e dissertações analisados constatou-se que as características do indivíduo são as principais causas utilizadas para justificar o encaminhamento por queixa escolar. Diz-se do estudante que ele possui déficit físico, cognitivo, emocional, neurológico e linguístico (Marçal, \& Silva, 2006). Do mesmo modo, diz-se da família que é pobre, desestruturada, que tem deficit cultural, social e educacional.

\section{A psicologia como mantenedora do fracasso escolar}

Sobre a atuação dos psicólogos que recebem alunos com queixa escolar, podemos afirmar que, na literatura consultada, esses profissionais adotam o modelo clínico não contextualizado, com foco nas questões emocionais e individuais. As crianças encaminhadas a esses serviços têm seu desempenho descrito a partir do registro de problemas de desenvolvimento e da perspectiva do contexto familiar imediato. Como afirmam Marçal (2005), Souza (2005) e Trautwein \& Nébias (2006), a causa da queixa escolar, na maioria das práticas psicológicas, é entendida como de caráter emocional.

A partir das críticas tecidas por alguns autores (Braga \& Morais, 2007; Neves \& Marinho-Araujo, 2006; Souza, 2005; Pereira, 2004), pode-se perceber que o encontro do psicólogo com as crianças tem funcionado como instrumento de diagnóstico e segregação, pois não realiza uma avaliação crítica das razões do encaminhamento. Marçal (2005) denuncia, ainda, a disparidade entre a clientela encaminhada pela queixa escolar e o sujeito idealizado da psicologia tradicional.

\section{A Escola como produtora do fracasso escolar}

Apesar de a escola ser um espaço de aprendizagem e formação, tem sido também palco de exclusão do aluno que não consegue aprender, o que a torna reprodutora do fracasso escolar. Em rigor, a escola não está constituída por um discurso que, por si só, seja crítico em relação à própria produção do fracasso. Este traço característico da prática institucional escolar tem muitos aspectos, os quais se expressam, por exemplo, na desvalorização do lugar do professor (tanto na sua formação como no seu reconhecimento social), na estigmatização das famílias (sobretudo daquelas em situação de vulnerabilidade socioeconômica), na ausência de espaços para reflexão entre educadores e pais e na falta de uma postura escolar baseada na escuta da criança.

Nakamura e cols. (2008) situam o sistema educacional como reprodutor de segregação e opressão, reforçando a visão foucaultiana de Bernardes (2008). Para esta última, na contemporaneidade há uma tendência a descrever os fenômenos psíquicos e sociais em uma linguagem médico-fisicalista, bem como uma crescente multiplicação de distúrbios e diagnósticos, aliada à medicalização dos supostos transtornos.

Nessa direção, Rosa (2011) afirma que há uma descaracterização da escola enquanto um espaço pedagógico, tornando-se ela cada vez mais um espaço clínico. Alguns autores (Braga \& Morais, 2007; Souza, 2005; Bernardes, 2008) argumentam que as escolas psicologizam o "não aprender", tornando o "aprender" uma noção exclusivamente neuropsicológica. Isto marca uma clara desapropriação do saber pedagógico e uma desresponsabilização no tocante ao fracasso escolar.

De acordo com Pereira (2004), as queixas escolares não incluem as práticas escolares, o que dificulta ou impede uma compreensão ampla e contextualizada do fracasso escolar. Com isso, são mantidos métodos de ensino antiquados, conteúdos descontextualizados e a divisão dos alunos em turmas consideradas fracas, médias e fortes, sem qualquer revisão do papel da escola na manutenção do fracasso.

De modo geral, a queixa escolar se refere a problemas emocionais, familiares e de comportamento. Segundo Scortegagna e Levandowski (2004), as queixas múltiplas 
são mais comuns nos encaminhamentos do que aquelas que fazem referência a apenas um único problema. A dificuldade de atenção e a de concentração são as queixas mais frequentes relacionadas aos problemas de aprendizagem, ao lado da dificuldade na aquisição da leitura e da escrita. As queixas relacionadas aos problemas de comportamento, na maioria das vezes, estão associadas à inquietação da criança, à desobediência às regras e à falta de limites.

\section{Considerações finais}

Em linhas gerais, nos últimos dez anos a queixa escolar vem sendo estudada, majoritariamente, numa perspectiva crítica e sócio-histórica, o que fica nítido nos achados desta revisão: das 21 publicações analisadas, 13 apresentam esse enfoque. Nesses trabalhos, frequentemente é apontada a necessidade de entendê-la de forma ampliada e crítica, lançando-se um novo olhar sobre a imputação de responsabilização exclusiva aos pais e aos alunos, incluindo questões escolares como possíveis produtoras e mantenedoras do fracasso escolar; entretanto, tal tendência não é verificada nas práticas de atendimento à queixa escolar, as quais apresentam, preponderantemente, um modelo clínico e descontextualizado, com pouco ou nenhum contato com a escola. Por outro lado, os estudos que adotam uma perspectiva cognitiva e comportamental, de modo geral, focalizam questões individuais e familiares relacionadas à queixa escolar, tais como diferenças de gênero, problemas emocionais, habilidades sociais e suporte parental.

A partir das contribuições trazidas por essas duas grandes abordagens teórico-metodológicas, percebe-se a importância de rever e criticar o discurso que culpabiliza o aluno e sua família pelo fracasso escolar, embora os autores não sugiram desconsiderar os elementos individuais e familiares. As questões neurológicas, de gênero, familiares, cognitivas e afetivas dos alunos existem e devem ser consideradas, mas não como causas únicas dos problemas escolares. Esses elementos devem ser entendidos numa complexa e dialética rede de relações e influências permeada por processos sociais, históricos, culturais, institucionais, políticos e econômicos, a qual, em sua integralidade, constitui a real determinante dos problemas de escolarização e da queixa escolar.

Nos trabalhos revisados a queixa escolar foi estudada mediante um recorte majoritariamente psicológico, em 17 dos 21 trabalhos. Com isso, pode-se ilustrar aquilo que chamamos de desapropriação do saber pedagógico relacionado à queixa. A escola e os fenômenos escolares são atravessados por uma terceirização do cuidado destinado aos profissionais de saúde. Bernardes (2008) sustenta, ainda, que a culpabilização dos alunos pelo fracasso escolar e seus supostos déficits constitui-se empecilho para o trabalho pedagógico, ideia que pode ser mais bem elucidada com a tese de Braga e Morais (2007), segundo a qual as escolas, tendendo a psicologizar e biologizar as queixas escolares, deixam de fazer o mais importante: reformular suas práticas pedagógicas e rever as questões relacionadas à instituição educacional.
Outra desigualdade na produção de estudos sobre a queixa escolar no Brasil, verificada nesta revisão, é a procedência regional dos estudos. Dos 21 trabalhos analisados, 15 procederam da região Sudeste, sobretudo do Estado de São Paulo, com doze trabalhos publicados. Talvez essa centralização decorra do fato de os cursos de Psicologia terem sido implementados, inicialmente, nessa região do país, onde inclusive está grande parte dos laboratórios e pesquisadores da área. Daí a importância de se fazerem novos estudos acerca da queixa escolar, os quais devem ser desenvolvidos principalmente em outros contextos regionais, a fim de que se enriqueça a discussão e se ampliem as referências conceituais e práticas em torno do tema.

\section{Referências}

Araújo, G. M. G. (2006). Implicações do perfil e das concepções dos Pedagogos, que atuam em equipes multidisciplinares da Secretaria de Estado de Educação do Distrito Federal, no atendimento às queixas escolares. Dissertação de Mestrado, Universidade de Brasília, Brasília.

Barros, S. K. S. N. (2008). Treinamento de habilidades sociais para pais de crianças com queixas escolares. Dissertação de Mestrado, Universidade Federal de São Carlos, São Carlos-SP.

Bernardes, M. G. M. (2008). Inventariando a produção do "alunoproblema": a queixa escolar em questão. Dissertação de Mestrado, Universidade de São Paulo, São Paulo.

Bock, A. M. B., \& Aguiar, W. M. J. (2003). Psicologia da educação: em busca de uma leitura crítica e de uma atuação compromissada. Em A. M. B. Bock, A perspectiva sócio-histórica na formação em psicologia (pp. 132-160). Petrópolis, RJ: Vozes.

Braga, S. G., \& Morais, M. L. S. (2007). Queixa escolar: atuação do psicólogo e interfaces com a educação. Psicologia USP, 18 (4), 35-51.

Bray, C. T., \& Leonardo, N. S. T. (2011). As queixas escolares na compreensão de educadoras de escolas públicas e privadas. Psicologia Escolar e Educacional, 15 (2), 251-261.

Cabral, E., \& Sawaya, S. M. (2001). Concepções e atuação profissional diante das queixas escolares: os psicólogos nos serviços públicos de saúde. Estudos de Psicologia, 6 (2), 143-155

D’Avila-Bacarji, K. M. G., Marturano, E. M., \& Elias, L. C. S. (2005). Suporte Parental: um estudo sobre crianças com queixas escolares. Psicologia em Estudo, 10 (1), 107-115.

Dazzani, M. V. M., \& Faria, M. O. (2009). Família, escola e desempenho acadêmico. Em Dazzani, M. V. M., \& Lôrdelo, J. A. (Orgs.), Avaliação Educacional: desatando e reatando nó (Vol. 1, pp 249-264). Salvador: EDUFBA. 
Dias, R. (2008). O atendimento psicológico a crianças com problemas escolares: a queixa escolar nos prontuários de psicologia. Dissertação de Mestrado, Pontifícia Universidade Católica de São Paulo, São Paulo.

Elias, L. C. S., \& Marturano, E. M. (2005). Oficinas de linguagem: proposta de atendimento psicopedagógico para crianças com queixas escolares. Estudos de Psicologia, 10 (1), 53-61.

Facci, M. G. D. (2004). A Periodização do Desenvolvimento Individual na Perspectiva de Leontiev, Elkonin e Vigotski. Cadernos Cedes, 24 (62), 64-81.

Ferreira, A. A., Conte, K. M. . \& Marturano, E. M. (2011). Meninos com queixa escolar: autopercepções, desempenho e comportamento. Estudos de Psicologia, 28 (4), 443-451.

Machado, A. M. (1997). A queixa escolar no alvo dos diagnósticos. Ideias, 28, 141-158.

Marçal, V. P. B. (2005). A queixa escolar nos ambulatórios de saúde mental da rede pública de Uberlândia: práticas e concepções dos psicólogos. Dissertação de Mestrado, Universidade Federal de Uberlândia, Uberlândia-MG.

Marçal, V. P. B., \& Silva, S. M. C. (2006). A queixa escolar nos Ambulatórios Públicos de Saúde Mental: Práticas e Concepções. Psicologia Escolar e Educacional, 10 (1), 121-131.

Marinho-Araujo, C. M., \& Guzzo, R. S. L. (Orgs.). (2011). Psicologia Escolar: Identificando e Superando Barreiras. Campinas: Editora Átomo \& Alínea.

Marturano, E. M., Toller, G. P., \& Elias, L. C. S. (2005). Gênero, adversidade e problemas socioemocionais associados à queixa escolar. Estudos de Psicologia, 22 (4), 371-380.

Moysés, M. A. A. (1998). A institucionalização invisível: crianças que não-aprendem-na-escola. Tese de Livre Docência, Universidade Estadual de Campinas, Campinas-SP.

Nakamura, M. S., Lima, V. A. A., Tada, I. N. C., \& Junqueira, M. H. R. (2008). Desvendando a queixa escolar: um estudo no Serviço de Psicologia da Universidade Federal de Rondônia. Psicologia Escolar e Educacional, 12 (2), 423-429.

Neves, M. M. B. J., \& Marinho-Araujo, C. M. (2006). A questão das dificuldades de aprendizagem e o atendimento psicológico às queixas escolares. Aletheia, 24, 161-170.

Nogueira, M. A. (2004). Favorecimento econômico e excelência escolar: um mito em questão. Revista Brasileira de Educação, 26, 133-144.

Nogueira, M. A. (2006). Família e escola na contemporaneidade: os meandros de uma relação. Educação e Realidade, 31, 155-169.
Nogueira, M. A., \& Abreu, R. C. (2004). Escola pública e famílias populares: uma relação dissonante. Educação em Revista, 39, 41-60.

Panaia, C. C. (2007). Crianças com queixa escolar: fatores de risco para desistência e abandono do atendimento psicopedagógico. Dissertação de Mestrado, Universidade de São Paulo, Ribeirão Preto-SP.

Patto, M. H. S. (1990). A produção do fracasso escolar: histórias de submissão e rebeldia. São Paulo: T. A. Queiroz.

Pereira, M. L. I. E. M. (2004). Projeto Prisma: uma alternativa de trabalho com crianças com queixa escolar. Dissertação de Mestrado, Universidade de São Paulo, São Paulo.

Rosa, A. P. (2011). Os bastidores da queixa escolar: da sala de aula ao consultório psicológico. Dissertação de Mestrado, Universidade Federal de Uberlândia, Uberlândia-MG.

Scortegagna, P.,souza \& Levandowski, D. C. (2004). Análise dos encaminhamentos de crianças com queixa escolar da rede municipal de ensino de Caxias do Sul. Interações, 9 (18), 127-152.

Souza, B. P. (2006). Orientação à queixa escolar: considerando a dimensão social. Psicologia: Ciência e Profissão, 26 (2), 312-319.

Souza, B. P. (Org.). (2007). Orientação à queixa escolar. São Paulo: Casa do Psicólogo.

Souza, M. P. R. (2000). A queixa escolar na formação de psicólogos. Em Tanamachi, E., Souza, M. P. R., \& Rocha, M. (Orgs.), Psicologia e Educação: desafios teórico-práticos. São Paulo: Casa do Psicólogo.

Souza, M. P. R. (2005). Prontuários revelando os bastidores do atendimento psicológico à queixa escolar. Estilos da Clínica, 10 (18), 82-107.

Souza, M. P. R. (2009). Psicologia Escolar e Educacional em busca de novas perspectivas. Psicologia Escolar e Educacional, 13 (1), 179-182.

Trautwein, C. T. G., \& Nébias, C. (2006). A queixa escolar por quem não se queixa - o aluno. Mental, 4 (6), 123-148

Zibetti, M. L. T. Souza, F. L. F., \& Queiróz, K. K. M. (2010). Quando a escola recorre à Psicologia: mecanismos de produção, encaminhamento e atendimento à queixa na alfabetização. Estudos e Pesquisas em Psicologia, 10 (2), 490-506. 
Recebido em: 15/03/2013

Reformulado em: 19/08/2013

Aprovado em: 28/08/2013

\section{Sobre os autores}

Maria Virgínia Machado Dazzani (dazzani@superig.com.br)

Doutora em Educação. Professora Adjunta do Instituto de Psicologia da Universidade Federal da Bahia.

Eliseu de Oliveira Cunha (eliseucunha@ig.com.br)

Graduando em Psicologia. Bolsista de Iniciação Científica da Universidade Federal da Bahia.

Polyana Monteiro Luttigards (polyluttigards@gmail.com)

Graduanda em Psicologia. Bolsista de Iniciação Científica da Universidade Federal da Bahia.

Patrícia Carla Silva do Vale Zucoloto (patriciavz@ig.com.br)

Doutora em Psicologia, Professora Adjunta da Faculdade Ruy Barbosa.

Gilberto Lima dos Santos (gilblimas@hotmail.con)

Doutor em Psicologia, Professor Adjunto, Universidade Estadual da Bahia.

Este estudo recebeu apoio financeiro do CNPq, Edital Universal MCTI/CNPq N. 14/2012. 